\title{
The dynamic landscape of fission yeast meiosis alternative-splice isoforms
}

\author{
Zheng Kuang, ${ }^{1,2}$ Jef D. Boeke, ${ }^{1,2}$ and Stefan Canzar ${ }^{3,4}$ \\ ${ }^{1}$ Institute for Systems Genetics, NYU Langone Medical Center, New York, New York 10016, USA; ${ }^{2}$ Department of Biochemistry \\ and Molecular Pharmacology, NYU Langone Medical Center, New York, New York 10016, USA; ${ }^{3}$ Toyota Technological Institute \\ at Chicago, Chicago, Illinois 60637, USA; ${ }^{4}$ Gene Center, Ludwig-Maximilians-Universität München, 81377 Munich, Germany
}

\begin{abstract}
Alternative splicing increases the diversity of transcriptomes and proteomes in metazoans. The extent to which alternative splicing is active and functional in unicellular organisms is less understood. Here, we exploit a single-molecule long-read sequencing technique and develop an open-source software program called SpliceHunter to characterize the transcriptome in the meiosis of fission yeast. We reveal 14,353 alternative splicing events in 17,669 novel isoforms at different stages of meiosis, including antisense and read-through transcripts. Intron retention is the major type of alternative splicing, followed by alternate "intron in exon." Seven hundred seventy novel transcription units are detected; 53 of the predicted proteins show homology in other species and form theoretical stable structures. We report the complexity of alternative splicing along isoforms, including 683 intra-molecularly co-associated intron pairs. We compare the dynamics of novel isoforms based on the number of supporting full-length reads with those of annotated isoforms and explore the translational capacity and quality of novel isoforms. The evaluation of these factors indicates that the majority of novel isoforms are unlikely to be both condition-specific and translatable but consistent with the possibility of biologically functional novel isoforms. Moreover, the co-option of these unusual transcripts into newly born genes seems likely. Together, the results of this study highlight the diversity and dynamics at the isoform level in the sexual development of fission yeast.
\end{abstract}

[Supplemental material is available for this article.]

Splicing is a fundamental process which removes intragenic noncoding regions (introns) and forms mature mRNAs for proper translation (Wang and Burge 2008; Lee and Rio 2015). It provides an important checkpoint and a posttranscriptional layer of gene expression control (Le Hir et al. 2003; McGlincy and Smith 2008; Braunschweig et al. 2013; Bentley 2014). One of the regulatory mechanisms is alternative splicing (AS), which leads to multiple transcripts from the same gene (Wang et al. 2008). AS is achieved mainly by different combinations of exons and introns or AS sites, producing a vast expansion of transcriptome diversity and, potentially, protein diversity (Keren et al. 2010; Nilsen and Graveley 2010). AS is considered a potent regulator of gene expression in multicellular organisms given the rationale that isoforms generated by AS are differentially regulated in different tissues or conditions (Wang et al. 2008). However, AS in unicellular organisms is less extensively explored. Important questions are: What is the complexity of single cell transcriptomes? How is AS differentially regulated across different conditions? Is AS even functionally relevant to eukaryotic microorganisms? Understanding these questions in unicellular organisms will extend our knowledge of AS and, in particular, the origin of AS.

Saccharomyces cerevisiae and Schizosaccharomyces pombe are the two most documented unicellular model organisms. S. cerevisiae has 280 intron-containing genes, and the vast majority of them have a single intron. On the contrary, $>2200$ genes in $S$. pombe are currently known to contain introns, and half of these genes have multiple introns. Therefore, $S$. pombe is a more suitable unicellular model to study AS and transcriptome diversity than $S$.

Corresponding authors: jef.boeke@nyumc.org, canzar@ttic.edu Article published online before print. Article, supplemental material, and publication date are at http://www.genome.org/cgi/doi/10.1101/gr.208041.116. cerevisiae. Thousands of AS events have been identified recently under various conditions in WT and mutant cells via different techniques such as short read RNA-seq and lariat sequencing (Awan et al. 2013; Bitton et al. 2015; Stepankiw et al. 2015). RNA metabolism kinetics, including synthesis, splicing, and decay rates have also been determined in vegetative fission yeast (Eser et al. 2016). A broad spectrum of AS types is observed, similar to multicellular organisms. These studies suggest prevalent AS in this unicellular organism. However, these findings are limited in defining isoforms, which represent the complete structure and sequence of transcripts. Characterizing isoforms is critical to predicting the effects on the encoded proteome. Whether these AS events are functionally relevant or condition-specific also remains poorly explored.

To address these questions, we exploited a single-molecule real-time (SMRT) sequencing technique based on the Pacific Biosciences (PacBio) platform (Eid et al. 2009) and developed SpliceHunter, a novel open-source software program to systematically explore the transcriptome in $S$. pombe. PacBio sequencing features very long reads, which are suitable for full-length cDNA sequencing. Recently, PacBio sequencing has been used to examine the transcriptome in multiple metazoan organisms, such as chicken and human, and multiple plants, and identified thousands of annotated and novel isoforms (Au et al. 2013; Sharon et al. 2013; Martin et al. 2014; Thomas et al. 2014; Tilgner et al. 2014; Dong et al. 2015; Minoche et al. 2015). To systematically

(C) 2017 Kuang et al. This article is distributed exclusively by Cold Spring Harbor Laboratory Press for the first six months after the full-issue publication date (see http://genome.cshlp.org/site/misc/terms.xhtml). After six months, it is available under a Creative Commons License (Attribution-NonCommercial 4.0 International), as described at http://creativecommons.org/licenses/ by-nc/4.0/. 
characterize isoforms and explore their differential regulations in fission yeast, we performed time-course isoform-level profiling during the meiosis of $S$. pombe. The transcriptome is dramatically reshaped by meiosis, and multiple meiosis-specific AS events have been observed (Mata et al. 2002; Wilhelm et al. 2008). In this study, we summarize the diversity and dynamics of the transcriptome at the isoform level during $S$. pombe meiosis and explore the conservation and translational potential of AS isoforms.

\section{Results}

\section{Experimental design, workflow and definition of AS types}

To explore the diversity and dynamics of alternative splicing in $S$. pombe, we collected six time points of WT cells during meiosis from 0 to $10 \mathrm{~h}$ at 2-h intervals (Supplemental Fig. S1). Upon nitrogen starvation, $S$. pombe is synchronized and enters the premeiotic $S$ phase. After $\sim 4$ $\mathrm{h}$, meiotic division begins and is completed by $\sim 6 \mathrm{~h}$. Spore maturation follows, and mature spores are usually observed after 8-10 h. Poly(A) ${ }^{+}$RNA was purified, reverse-transcribed to cDNA, and sequenced with a PacBio sequencer. Five SMRT cells were used for two RNA replicates of each time point. Raw reads were clustered and polished using the Iso-Seq pipeline, and high-quality (HQ), full-length (FL), polished consensus sequences (referred to here as Iso-Seq reads) were output (Rhoads and $\mathrm{Au}$ 2015). Replicates were pooled for downstream analysis. We developed SpliceHunter, a novel software program that detects, quantifies, and compares complex splicing patterns along novel isoforms inferred from Iso-Seq reads. SpliceHunter reports the number of supporting reads of isoforms per sample or time point and predicts protein sequences. Based on the Iso-Seq algorithm, each Iso-Seq read is associated with multiple FL CCS (circular-consensus) reads and non-FL CCS reads. FL CCS reads are defined by co-existence of $5^{\prime}$ and $3^{\prime}$ adaptors and poly $(\mathrm{A})^{+}$tail. Therefore, we use the number of FL CCS reads as a proxy for abundance and dynamics analysis.

After mapping reads to a reference genome, SpliceHunter assigns reads to annotated or novel transcription units (TUs) (Fig. 1A, step 1) according to well-defined and adjustable criteria. After collapsing compatible reads to isoforms, each isoform is compared to the previously annotated exon-intron structure to detect AS events (Fig. 1A, steps 2, 3), antisense, and read-through transcripts. Finally, the effect of AS events on the encoded protein sequence is analyzed (Fig. 1A, step 4), including their combined shift in the open reading frame (ORF). We consider the following types of AS events (Fig. 1B): exon skipping, exon inclusion, intron retention, intron in exon, alternative acceptors and donors, and novel exons. See Methods for a formal definition of AS types and a detailed description of software tool SpliceHunter.

\section{Characterization of PacBio reads}

In total, we obtained $2,266,791$ CCS reads, and 1,311,840 of those were FL CCS reads (Fig. 2A; Supplemental Table S2). Through the Iso-Seq pipeline, 424,511 Iso-Seq reads were generated. The average length of CCS reads was $1285 \mathrm{bp}$, which was slightly longer than previous reports (Fig. 2B; Sharon et al. 2013; Thomas et al. 2014; Tilgner et al. 2014). The average length of FL CCS reads and Iso-Seq reads were 1094 and 1178 bp, respectively, and the length distributions did not vary across time points (Supplemental Fig. S2A). Six thousand one hundred ninety-nine S. pombe genes ( $\sim 90 \%$ of all genes) were recovered from PacBio sequencing. Median coverage of FL CCS reads per gene was 71, and many genes had coverage $>100$, which enabled deep discovery of novel isoforms (Fig. 2C). Pearson correlation coefficients of read counts between replicates ranged from 0.93 to 0.96 at different time points (Supplemental Fig. S2B), suggesting high technical reproducibility in our study. Additionally, FL CCS read counts showed strong correlation with expression levels inferred from Illuminabased short-read RNA-seq data despite variations in strains, conditions, and techniques (Supplemental Fig. S2C). This high correlation supports the reliability of our time course analysis, which is based on large differences between PacBio read counts at different time points and smooth trends. With respect to PomBase v2.29 definitions of (non-)coding RNA and pseudogenes, 4993

\section{Genome Research}

www.genome.org 
A

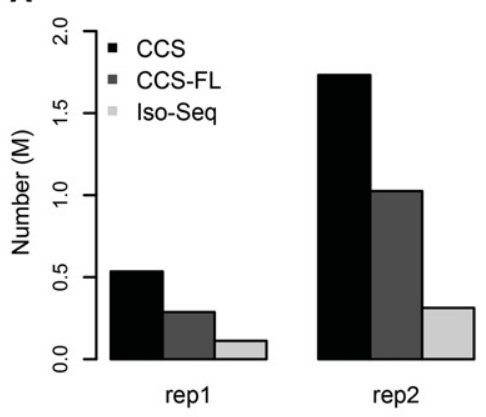

E

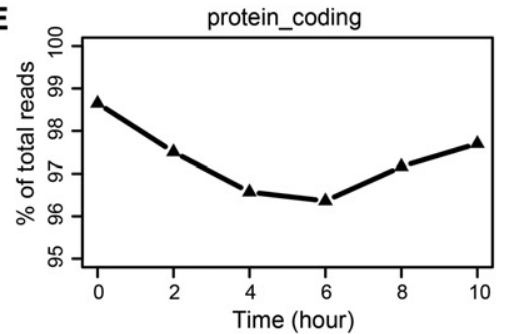

F

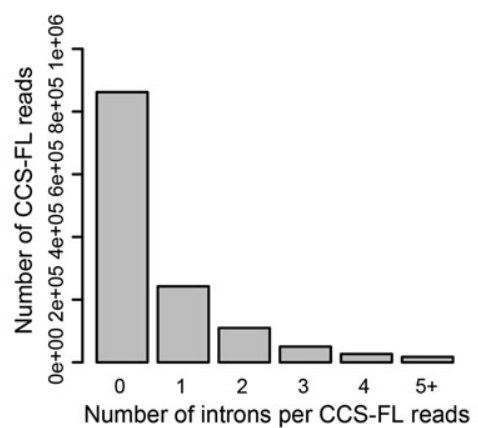

B
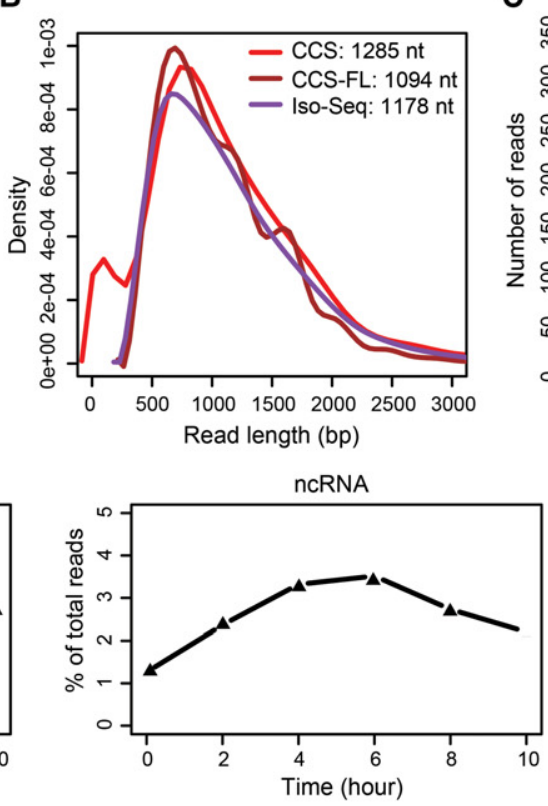

G

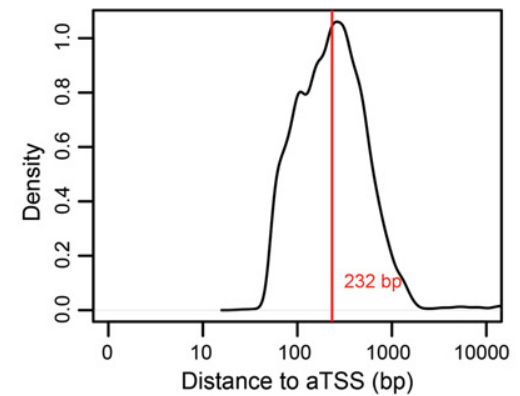

C

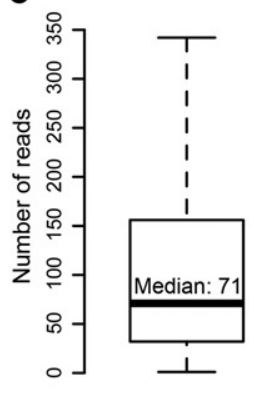

D

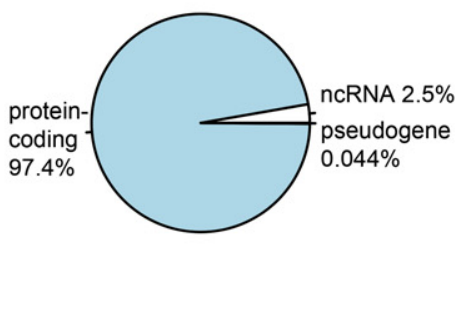

pseudogene

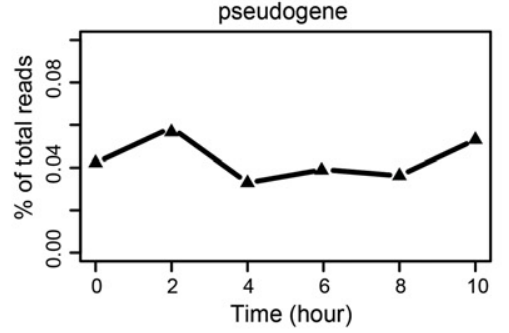

H

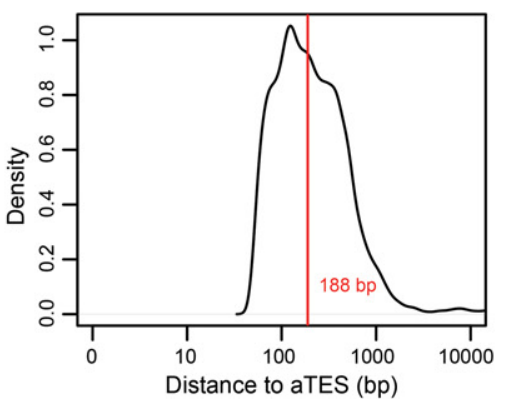

Figure 2. General properties of PacBio reads. (A) Numbers of CCS reads, full-length CCS reads (CCS-FL), and Iso-Seq reads from two replicates. The second batch has $4 \times$ size of the first batch. (B) Length distribution of CCS, CCS-FL, and Iso-Seq reads. (C) Box plot showing the $25^{\text {th }}, 50^{\text {th }}$, and $75^{\text {th }}$ percentiles of the numbers of FL CCS reads for each gene. Whiskers represent 1.5 inter-quartile range. (D) Pie plot showing the percentage of FLCCS reads corresponding to protein-coding genes, noncoding RNA genes, and pseudogenes. (E) Overall trends of FL CCS reads matching protein-coding genes, noncoding RNA genes, and pseudogenes. $(F)$ Counts of FL CCS reads with different numbers of introns. (G) Distribution of distances between the $5^{\prime}$ end of reads to the annotated TSS sites (aTSSs). Reads only outside the annotated TSS sites were counted. (H) Distribution of distances between the $3^{\prime}$ end of reads to the annotated TES sites (aTESs). Reads only outside the annotated TES sites were counted.

(97.1\%) protein-coding genes, 1121 (73\%) noncoding RNA (ncRNA) genes, and 18 (62.1\%) pseudogenes were recovered. Of the FL CCS reads, $97.4 \%$ were assigned to protein-coding genes which represented $80 \%$ of all captured genes, indicating higher coverage for protein-coding genes (Fig. 2D). Interestingly, the ratio of RNA molecules corresponding to protein-coding genes versus ncRNA decreased in the middle of meiosis and increased at the late stage (Fig. 2E). We further explored the complexity of PacBio reads by examining the number of introns per read. Although the majority of reads contained only 0 or 1 intron, there were still 190,101 reads with multiple introns, together providing substantial data to explore alternative splicing (Fig. 2F). Lastly, we explored the distances from the $5^{\prime}$ or $3^{\prime}$ end of reads to the corresponding gene transcription start site (TSS) or transcription end site (TES). We focused on reads which extended beyond the annotated TSS or TES because the SMARTer CDNA preparation method does not select for 7-methylguanosine $(7 \mathrm{mG})$ which marks intact $5^{\prime}$ end mRNA molecules. On average, reads extend 232 bp upstream of $5^{\prime}$ ends (Fig. 2G) and 188 bp downstream from $3^{\prime}$ ends (Fig. 2H) of annotated genes, suggesting alternative $5^{\prime}$ and 3' UTRs.

\section{Various types of alternative splicing detected in S. pombe meiosis}

Next, we explored AS isoforms in $S$. pombe. Although multiple studies have revealed different AS events, this is to our knowledge the first study to explore AS at the isoform level. Not surprisingly, we unveiled complex cases of alternative splicing. For example, we observed 59 distinct polyadenylated mRNA isoforms for SPAC12B10.05(icp55), containing different types of AS events either in the same or different isoforms (Fig. 3A). The capacity to detect multiple AS events in the same molecule by PacBio sequencing avoids underestimating isoform complexity.

Alternative splicing comes in many potential forms, and we realized that a formal nomenclature/classification scheme was needed to unambiguously discuss the many events we observed. Thus, we developed a rigorous classification scheme and nomenclature that embraces commonly known instances such as exon 
A

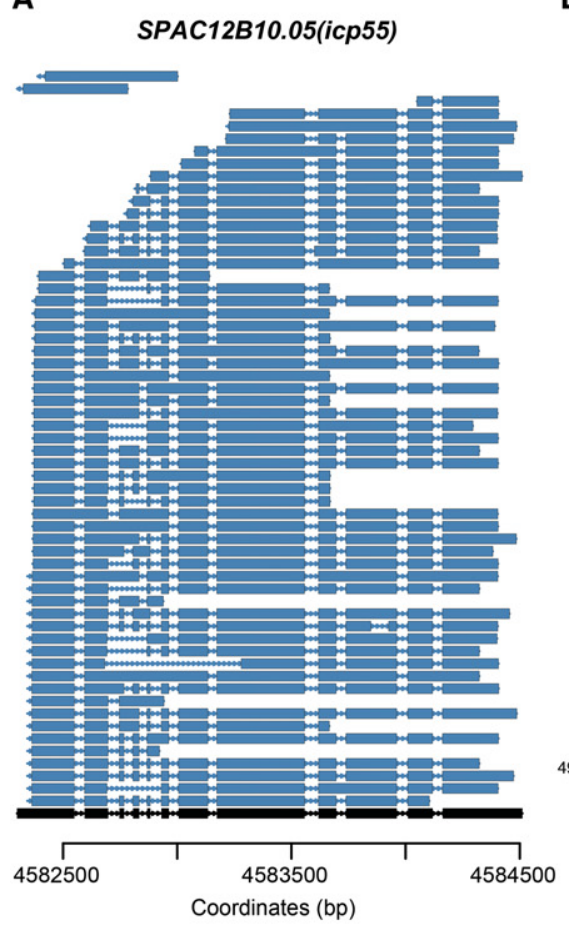

B
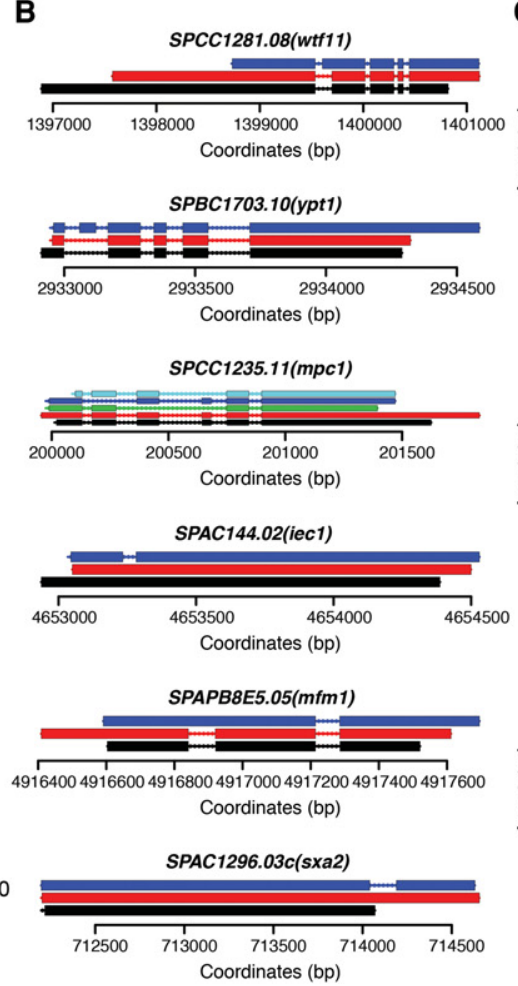

C
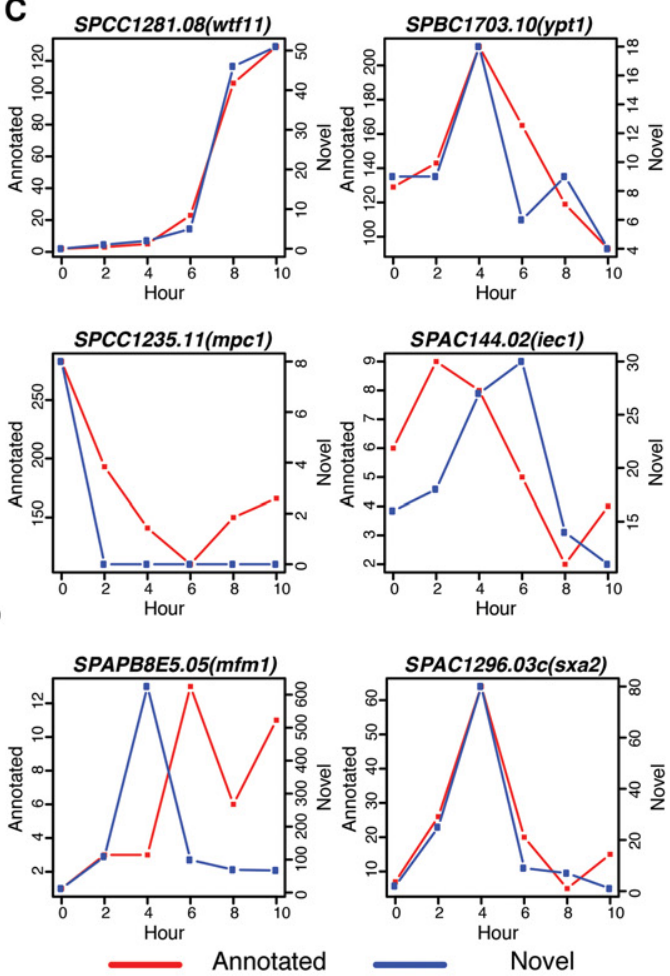

Figure 3. Examples of alternative splicing during S. pombe meiosis. (A) Fifty-nine different isoforms were detected for SPAC12B10.05(icp55). Black represents the annotation and dark blue represents detected isoforms. Aligned nucleotides are denoted by solid lines and alignment gaps indicating introns are marked by thin lines with arrows indicating the strand. (B) Six different types of alternative splicing structures. SPCC1281.08(wtf1 1) (novel splicing donor or acceptor); SPBC1 703.10(ypt1) (exon inclusion); SPCC1235.11(mpc1) (exon skipping); SPAC144.02(iec1) (intron in exon); SPAPB8E5.05(mfm1) (intron retention); SPAC1296.03c(sxa2) (novel exon). Black marks the annotated structure; red depicts isoforms that match the internal annotated structure; all other colors (dark blue, light blue, green) denote structurally different AS isoforms. (C) Dynamics of annotated isoform and novel isoform for each of the six examples shown in B. Red lines represent numbers of FL CCS reads for annotated isoforms and blue lines represent numbers of FL CCS reads for novel isoforms during meiosis.

skipping, intron inclusion, etc. Based on the definitions implemented in our algorithm (see Methods), we observed examples for all types of AS in $S$. pombe: an alternative splicing acceptor in SPCC1281.08(wtf11), an exon inclusion in SPBC1703.10(ypt1), multiple exon skipping events in SPCC1235.11(mpc1), an intron in exon in SPAC144.02(iec1), an intron retention in SPAPB8E5.05 (mfm1), and a novel exon in SPAC1296.03c(sxa2) (Fig. 3B). Interestingly, some of the novel isoforms had comparable or even more numerous supporting reads than the corresponding annotated isoforms (Fig. 3C). This implies that these isoforms were unlikely to represent rare erroneous byproducts that escaped mRNA surveillance. Although many novel isoforms showed splicing patterns well-correlated with the corresponding annotated isoforms, some novel isoforms exhibited distinct temporal patterns compared to the corresponding annotated isoforms (Fig. 3C), suggesting that the novel isoforms could be temporally differentially regulated. More examples are shown in Supplemental Figure S3.

\section{Landscape of alternative splicing}

After examining individual examples, we next sought to explore the landscape of AS and isoforms (Supplemental Table S1). The dominant type of AS event observed in S. pombe was intron retention, probably caused by a bypass of individual splicing sites (Fig. 4A; Supplemental Table S3). The retained introns are distributed similarly in length to the total of all annotated introns (Fig. 4G).
The next dominant type was "intron in exon," which can be considered the opposite of intron retention. Other types of AS events were observed but less abundant, including novel splicing sites, novel exon, and exon skipping/inclusion. Next, we asked how many genes have multiple isoforms. Interestingly, only $\sim 1300$ genes have a single detected isoform in this study and 1432 genes have two isoforms with a minimum of one FL CCS read per isoform (Fig. 4B). More than 3000 genes have $>2$ isoforms, suggesting pervasive complexity of the $S$. pombe transcriptome and potential AS-mediated regulation. Isoform counts per gene were further analyzed by increasing the minimum number of FL CCS reads required to support an isoform (Supplemental Table S4). Additionally, for the first time we are able to distinguish isoforms with single AS events (8739 or $77.8 \%$ ) and mRNA isoforms with multiple AS events (22.2\%) (Fig. 4C). To examine the molecular association of AS events, we examined 1708 pairs of alternatively retained introns (see Methods), the most abundant type in this study. We found 683 significantly dependent intron pairs at an FDR of 0.05 using Fisher's exact test. To quantify the association, we adopt the intragenic molecular association score previously defined (Tilgner et al. 2015), which is the ratio of the number of reads that retained both introns or skipped both introns to the total number of reads spanning both introns. Associated intron pairs show higher scores, indicating that their retention is co-associated rather than mutually exclusive (Fig. 4J). Only two pairs of the reverse type of event, novel introns in exons, were alternatively

\section{Genome Research}

www.genome.org 
A

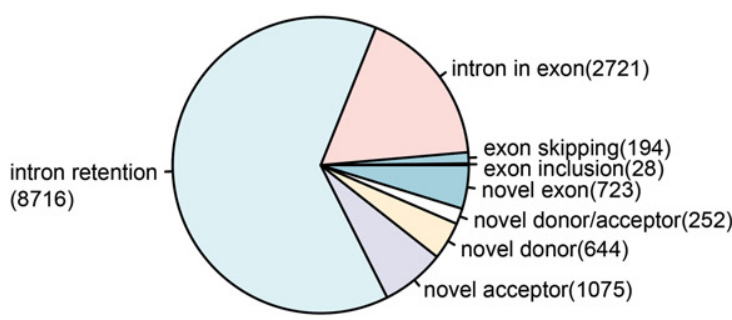

B

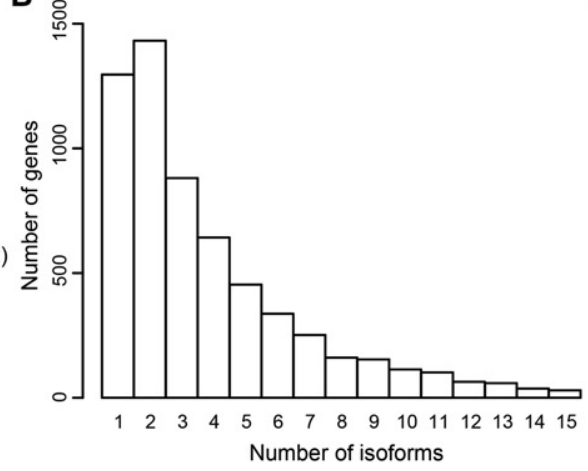

C

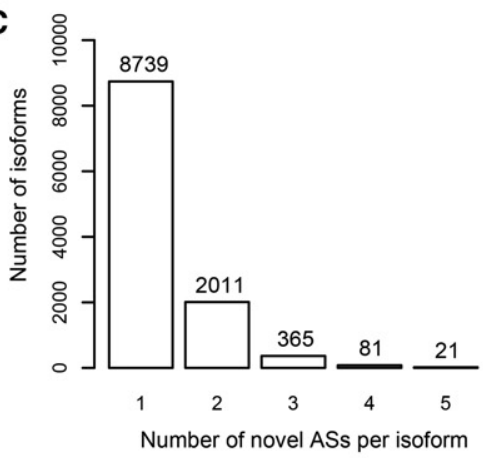

D

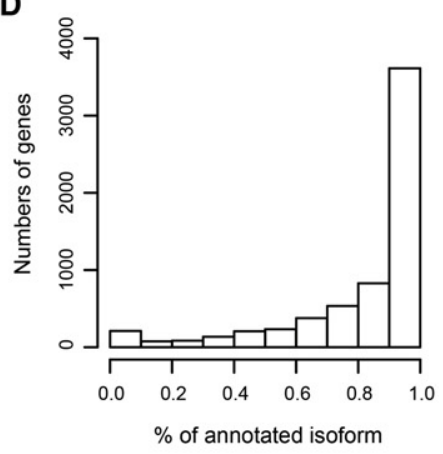

E

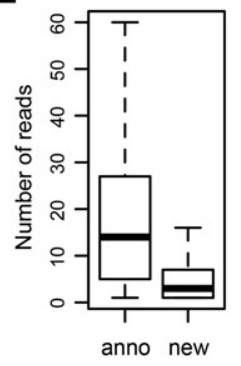

F

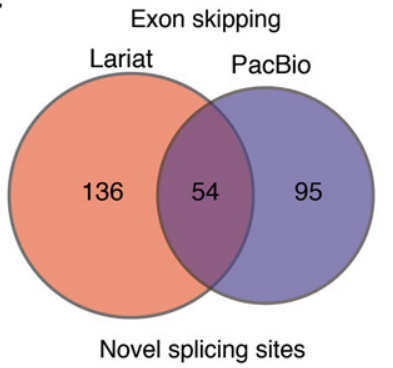

Lariat

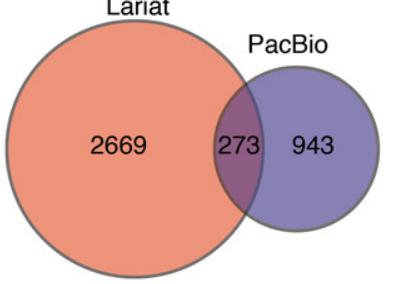

Novel intron

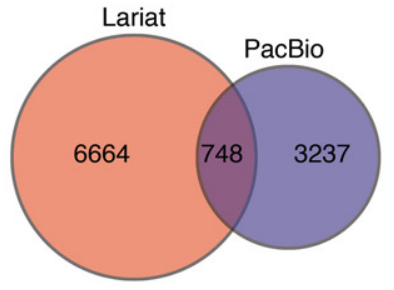

Canonical

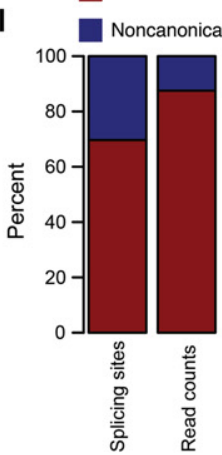

J

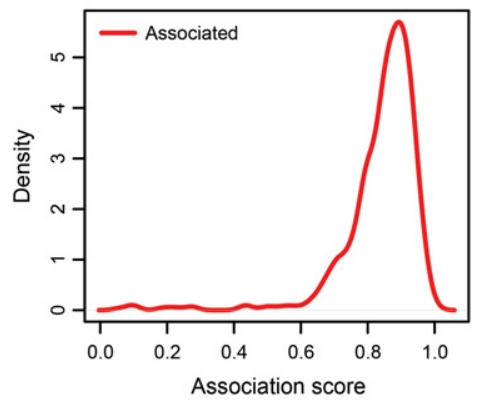

G

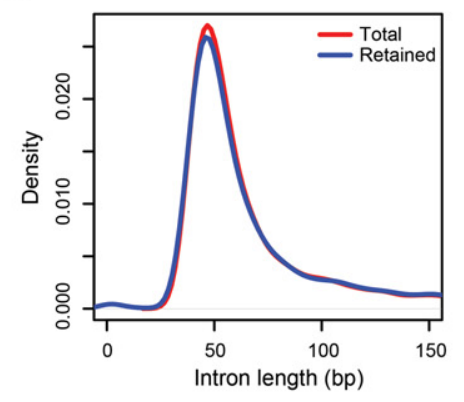

H

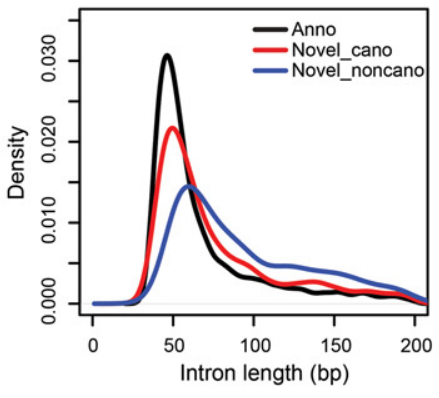
然 
dinucleotides at the novel splicing sites. At annotated splicing sites, $99.94 \%$ of the dinucleotides are GU-AG, with only three exceptions. However, GU-AG only appeared in $69.67 \%$ of novel splicing sites or in $87.5 \%$ of reads with novel introns (Fig. $4 \mathrm{I}$ ) after filtering for high-confidence alignments (see Methods). Neither the dinucleotides nor the full $5^{\prime}$ hexamers from noncanonical novel introns show any consensus sequence (Supplemental Fig. S4F), implying that many of these noncanonical introns might be aberrantly spliced (Supplemental Table S7). Intriguingly, novel introns with canonical splicing sites have similar lengths as annotated introns, but novel introns with noncanonical splicing sites are generally longer (Fig. $4 \mathrm{H}$ ). Pairs of dinucleotides with small hamming distances to GU-AG occur more often, such as 75 pairs of GU-UG, 55 pairs of GU-GG, etc.

Interestingly, we discovered some read-through transcripts that spanned two consecutive genes on the same strand, 57 of which contained annotated introns from both genes (Supplemental Tables S1, S8). Among read-through transcripts with introns, 14 had supporting reads more abundant than at least one of the individual transcripts, suggesting that these were less likely to represent chimeric reads. This is consistent with a previous finding of widespread "polycistronic" transcripts in fungi (Gordon et al. 2015). Supplemental Figure S4 shows four examples of readthrough transcripts, which are further discussed in the Supplemental Material.

Different methods have been applied in $S$. pombe to identify alternative splicing, including short-read RNA-seq, ribosomal profiling, and lariat sequencing (Awan et al. 2013; Duncan and Mata 2014; Bitton et al. 2015; Stepankiw et al. 2015). Here, we compared our results with corresponding events predicted from short-read RNA-seq, lariat sequencing, and ribosomal profiling. Fifty-five percent of skipped exons and 38\% of novel introns/splice sites reported in this study were also identified by short-read RNA-seq (Supplemental Fig. S4E; Bitton et al. 2015). Ribosomal profiling was performed in meiosis, too (Duncan and Mata 2014), and with the exception of novel start and stop codons, which were not predicted by our study, the remaining eight new isoforms detected were all validated in our study. Lariat sequencing was done in a $d b r 1 \Delta$ mutant strain from log phase, diauxic shift, and heat shock conditions (Stepankiw et al. 2015). The occurrences of three types of AS events were compared, including exon skipping, novel splicing sites, and novel intron. Surprisingly, all three types of AS events showed little overlap between the two data sets (Fig. 4F), presumably because the conditions in the two studies were distinct and the techniques captured different RNA molecules. This comparison suggests that the true complexity of AS in $S$. pombe is even higher than thus far reported.

\section{Dynamics of AS during meiosis}

When we examined AS events, we noticed that many alternative isoforms exhibited unexpected temporal patterns compared to annotated isoforms. To explore the dynamics of AS isoforms, we first summarized the general dynamic patterns of different types of AS events by examining the number of isoforms and number of reads supporting each type of AS. Most types of AS increased in meiosis (Fig. 5A), which was likely to be at least partially related to decreased RNA surveillance. However, exon-skipping events decreased at early stages of meiosis and increased at the late stage; intron retention was relatively unchanged. The dynamics of exon skipping suggest a condition-driven alternative usage of different exons between mitosis and meiosis.
Next, we examined isoform dynamics at the gene level. The abundance of the majority of individual AS isoforms was also increased during meiosis, consistent with the general patterns (Fig. 5B). We hypothesized that if AS is functionally associated with different conditions, the two isoforms for the same gene are more likely to be uncorrelated or anti-correlated. Therefore, Pearson's correlation coefficients were calculated between the number of reads supporting annotated and alternative isoforms for genes with comparable abundance of each isoform. A left-skewed histogram of coefficients (Fig. 5C; Supplemental Table S9) suggests that the majority of alternative isoforms were correlated in abundance with annotated isoforms. However, there were still 519 genes with anti-correlated isoforms and 104 genes with correlation $\leq-0.5$. To explore the anti-correlated isoforms, we selected 28 genes that were supported by more than 100 FL CCS reads for further analysis. The temporal patterns of novel and annotated isoforms were compared in a heat map (Fig. 5D). Some genes had alternative isoforms expressed in mitosis and annotated isoforms expressed in meiosis, whereas a second cluster of genes had annotated isoforms expressed in mitosis and switched to alternative isoforms in late meiosis.

SPAC30.03c (tsn1) encodes a translin protein (Laufman et al. 2005) and is up-regulated in middle and late meiosis (Mata et al. 2002; Martín-Castellanos et al. 2005). Interestingly, besides an increment of annotated isoform expression in middle to late meiosis, we also observed a 48-bp intron retention isoform expressed in early meiosis (Fig. 5E,F). SPAC15E1.08 (naa10) encodes an N-acetyltransferase catalytic subunit, which is essential for survival, and mutations in the $S$. cerevisiae homolog ARD1 cause sporulation defects (Mullen et al. 1989). The abundance of the novel isoform, which has a novel exon and an intron upstream of the annotated start codon, increased in late meiosis. It is possible that the new 5' UTR is involved in the gene's expression regulation or provides new start sites. A few examples were further examined in Supplemental Figure S5.

\section{Prediction of novel proteins}

One major role of alternative splicing is to generate distinct functional proteins from the same gene. On the contrary, a product of aberrant splicing is not responsible for generating functional proteins. Therefore, we performed systematic prediction of protein sequences from all isoforms detected in our study (Supplemental Table S10). Translation prediction always started from the annotated start codon if available and stopped at the first stop codon. By this definition, 18,166 isoforms were predicted to be translatable, while the remaining isoforms either belonged to genes that do not encode proteins or lacked the stop codon or annotated start codon (Fig. 6A). About 1200 of these lack the annotated start codon, and all but one of these contain at least one alternate ATG and a stop codon in the same frame and thus might in principle also encode functional proteins, but these isoforms were not studied further.

From 4990 genes, 14,292 distinct translatable sequences were predicted. Among these genes, 2116 genes were predicted to generate exclusively annotated proteins and 2852 genes were predicted to encode both annotated and novel protein sequences. Interestingly, for 22 genes, we observed a substantial number of reads supporting AS isoforms that encode protein sequences different from the annotated sequence, while the annotated isoform was not supported by any read (Supplemental Table S11). If not misannotated, these isoforms are potentially expressed at a very low level.

\section{Genome Research}

www.genome.org 
A
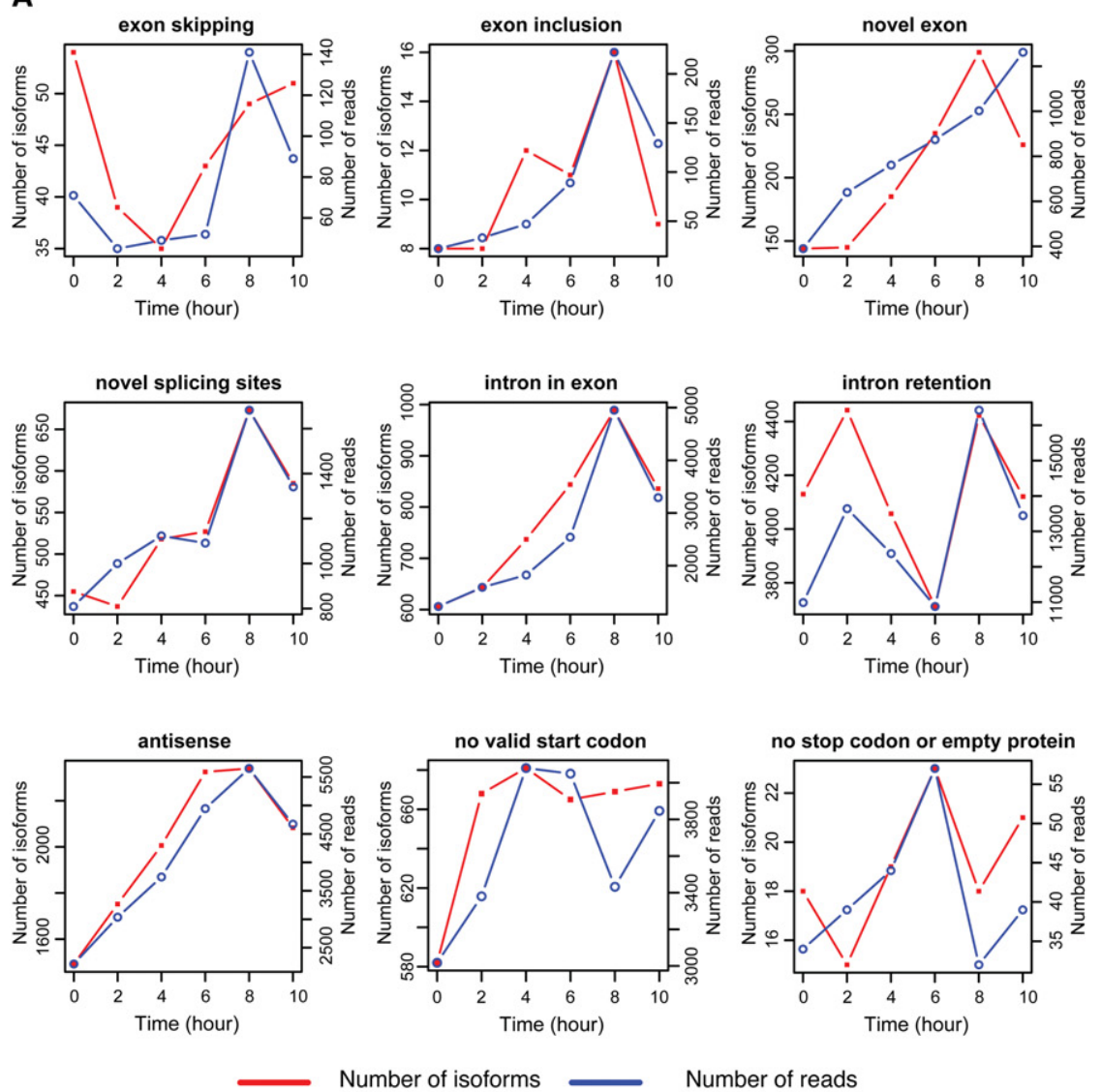

B
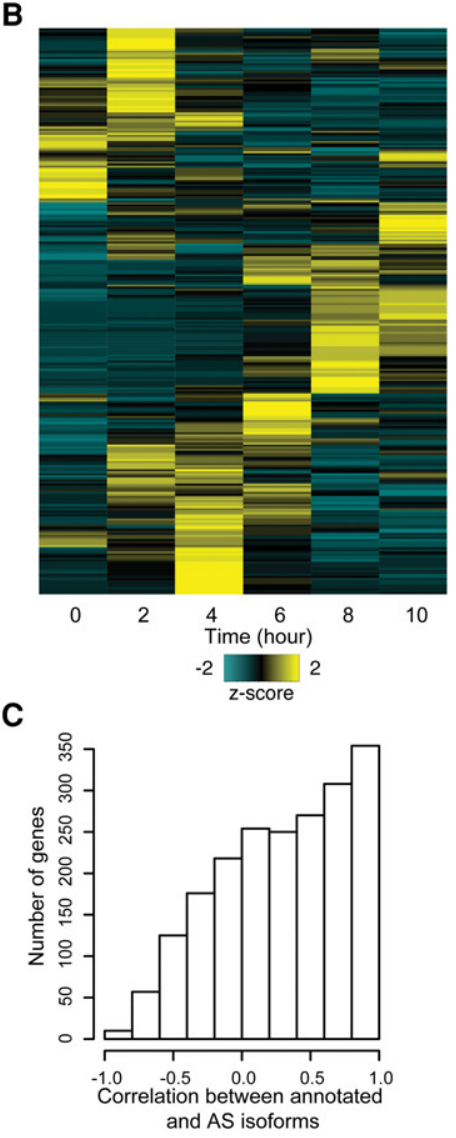

D

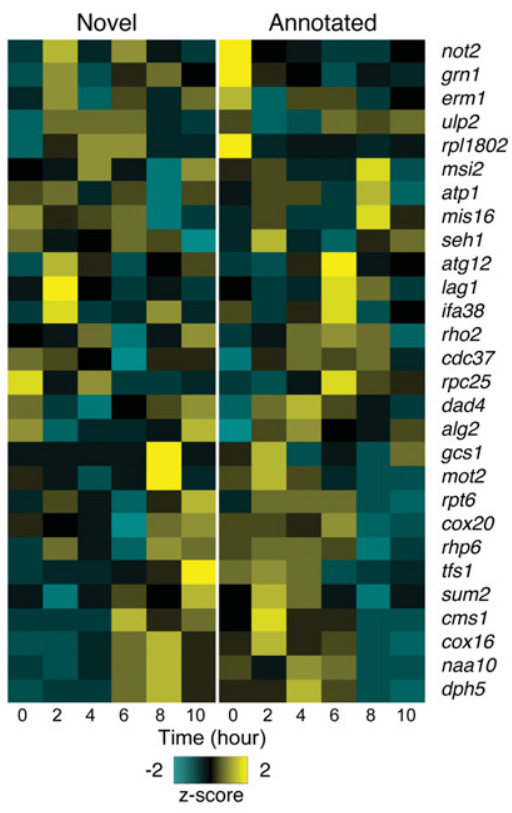

E

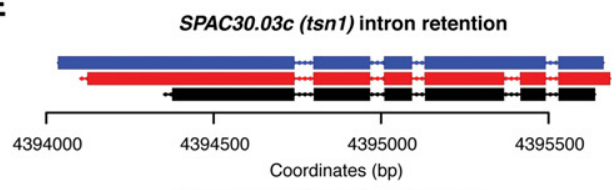

SPAC15E1.08 (naa10) novel exon

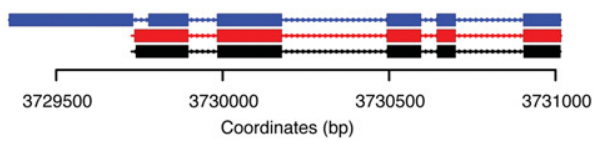

$\mathbf{F}$
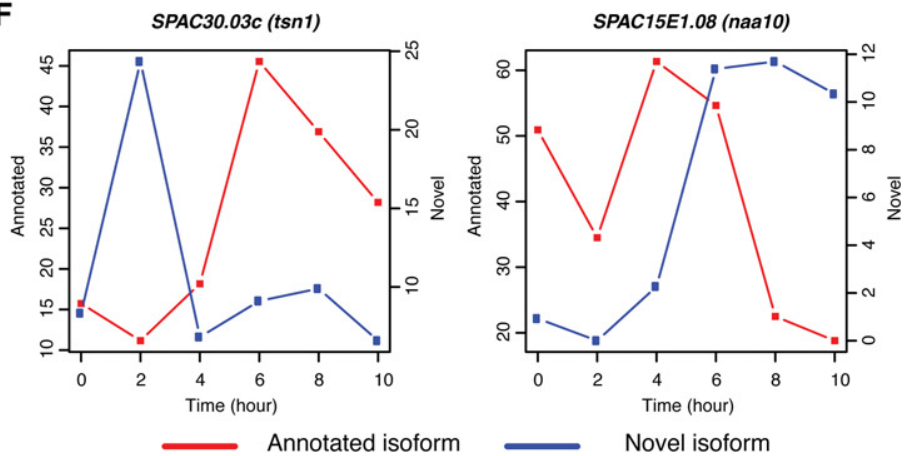

Figure 5. Dynamics of alternative splicing during meiosis. (A) Trends of different types of alternative splicing during meiosis. Red lines represent numbers of isoforms and blue lines represent numbers of FL CCS reads. (B) Heat map showing the temporal pattern of novel isoforms. (C) Histogram showing the correlation between annotated and alternative isoforms during meiosis. (D) Heat map showing the temporal pattern of novel and annotated isoforms. Genes with correlation smaller than -0.4 , FL CCS read count $>100$, and percentage of annotated isoform $<90 \%$ were chosen for hierarchical clustering. $(E, F)$ Examples of anti-correlated isoforms in meiosis. Panel $E$ shows the exon-intron structures of the examples; panel $F$ shows the temporal patterns of the annotated and alternative isoforms. 


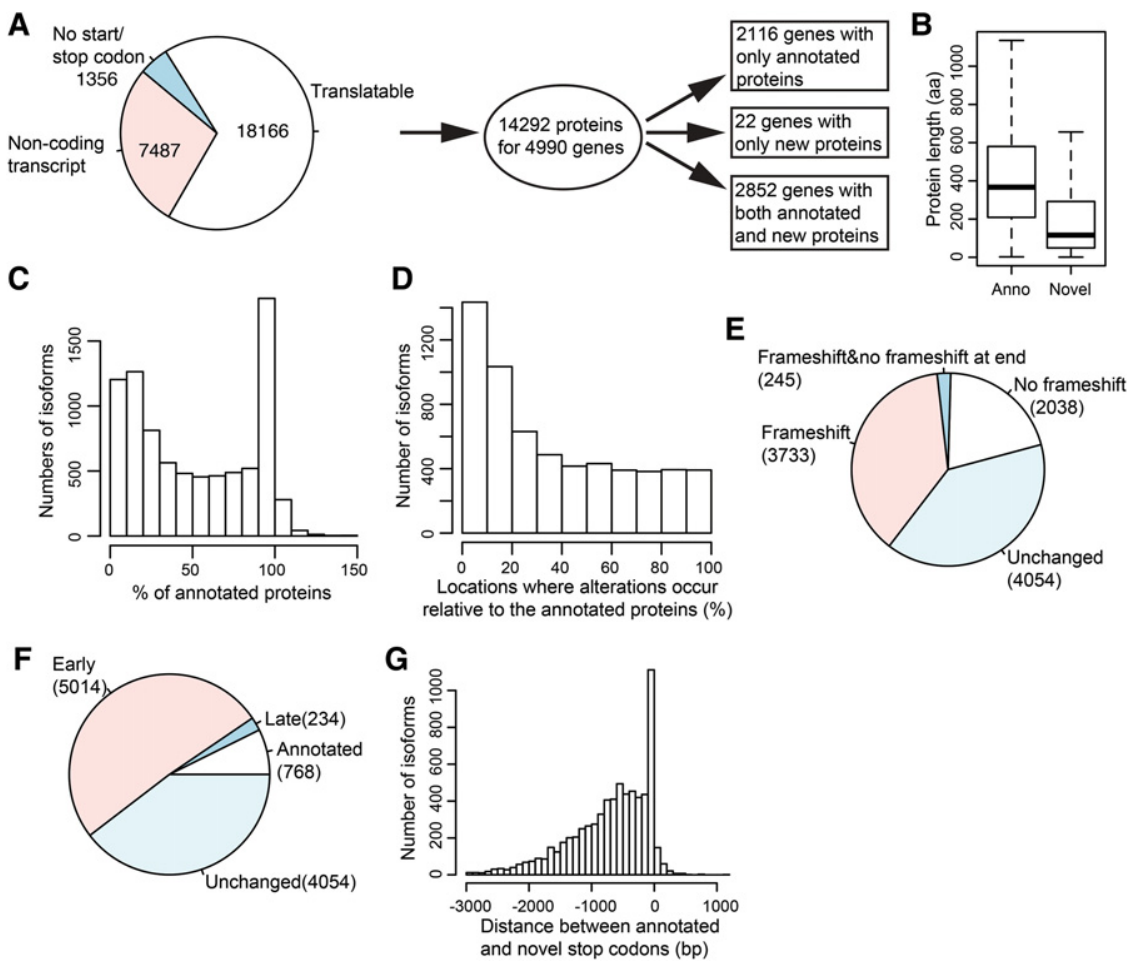

Figure 6. Predicting translational products from detected isoforms. (A) Summary of protein prediction. The left pie plot shows the number of translatable isoforms. From 4990 genes, 14,292 protein sequences were predicted, and these genes were further broken up by the status of annotated and new protein sequences. (B) Box plot indicating the length of annotated and novel protein sequences. (C) For isoforms encoding novel protein sequences, the histogram shows the ratio of the length of novel protein sequences relative to the length of the corresponding annotated protein sequence. $(D)$ Histogram showing where alteration occurs in the predicted protein relative to the corresponding annotated protein. (E) Fractions of translatable isoforms with novel AS events which contain frameshift or not. Unchanged refers to AS isoforms with the same protein sequence as the annotated one. $(F)$ Fractions of translatable isoforms with novel AS events which have early, late, or annotated stop codons. $(G)$ Histogram showing the distances between annotated and novel stop codons for isoforms with novel predicted proteins.

A predicted novel protein may not actually be produced. We sought to theoretically address this question by quantifying the impact of alternative splicing on the protein level with respect to the annotated sequence. We examined important protein sequence properties, including its length, relative position of protein sequence alteration caused by AS, how it affects the reading frame, and where the hypothetical translation of novel proteins terminates compared to the corresponding annotated proteins. In general, novel protein sequences were shorter than annotated protein sequences (Fig. 6B). We observed a major mode at isoforms which encode novel protein sequences with lengths similar to annotated proteins and a minor mode at isoforms which encode novel proteins with $<20 \%$ of annotated protein length (Fig. 6C; Supplemental Fig. S6D). Furthermore, the alteration in predicted protein sequences due to alternative splicing usually occurs at the beginning of a coding sequence (Fig. 6D; Supplemental Fig. S6E). Overall, $40.3 \%$ of translatable isoforms with AS events encoded the same proteins as annotated isoforms. More than half of the remaining isoforms which encode novel protein sequences are at least partially not in the same reading frame as the corresponding annotated isoform (Fig. 6E). Furthermore, $\sim 33.9 \%$ of isoforms encoding novel protein sequences were in the same reading frame as annotated isoforms and another $\sim 4.07 \%$ of isoforms had alterations that shifted the reading frame followed by a second al- teration that restored the frame. Furthermore, $47.9 \%$ of AS isoforms had translation ending at the annotated stop codons (Fig. 6F). Only for $2.32 \%$ of AS isoforms, translation ended downstream from, but close to, annotated stop codons (Fig. 6G). Most isoforms with altered protein sequences (49.8\%) showed early translation termination with varying distances between annotated and novel stop codons. We call a stop codon novel if it terminates the predicted translation of AS isoforms at a different location than the annotated stop codon.

In addition, we attempted to predict protein-coding regions for the 770 new TUs. The protein length was generally very short with a median length of $\sim 30$ aa; the longest was $>200$ aa (Supplemental Fig. S6A-C). The majority of novel TUs had no ORF longer than $25 \%$ of their full-length sequence. Previous work has hypothesized that some or most ncRNAs represent transcriptional noise (Hüttenhofer et al. 2005; Costa 2007) but may also represent fodder for the birth of new genes (Carvunis et al. 2012). This is supported by the observation that only $13 \%$ of the novel TUs we identified were shown to bind to ribosomes in a previous ribosomal profiling study (Duncan and Mata 2014).

\section{Protein sequence conservation and secondary structure}

Following the protein prediction analysis, we next sought to check the quality of these novel protein sequences by evaluating their conservation and secondary structure formation. Two major classes of novel protein sequences were examined. Hypothetical proteins predicted from novel TUs and novel C-terminal amino acid sequences starting at the first alteration in AS isoforms. Conservation of novel sequences was assessed by searching for sequence-similar proteins in fission yeasts, fungi, and eukaryotes using BLAST (Gish and States 1993). Secondary and tertiary structures and their properties were predicted by RaptorX (Källberg et al. 2012). For 53 novel TUs, we found evidence for homology in other species with less than 100 matching amino acids (Supplemental Fig. S7C), indicating their potential translation. The majority of them had homologs in other related fission yeasts without significant preference (Fig. 7A; Supplemental Table S12). For example, the predicted protein of NG_554 highly resembles a membrane transporter such as allantoate permease in other fission yeasts, and it could form a structure with $64 \% \alpha$-helix (Supplemental Fig. S7A). The longest protein sequence predicted from a novel TU (NG_569) is similar to retrotransposons in different fission yeasts (Supplemental Fig. S7B). Additionally, we examined conservation of C terminus novel amino acid sequences from 550 AS isoforms which have $>2$ CCS FL supporting reads and are at least 19 aa long. One hundred seventy-seven AS isoforms have homologs in other species (Fig. 7B; Supplemental Table S12).

\section{Genome Research}

www.genome.org 
A

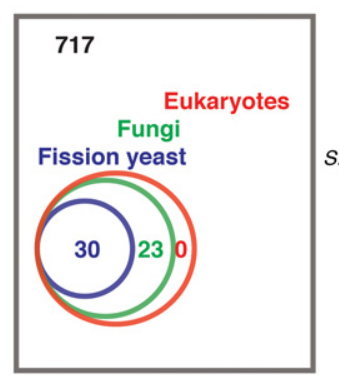

C.

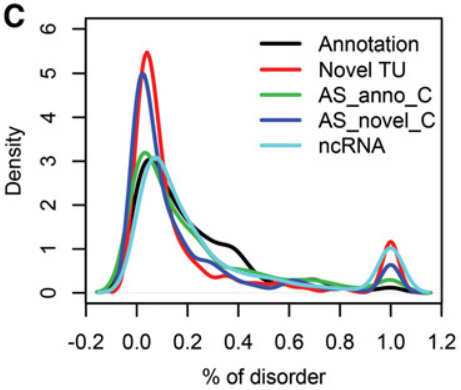

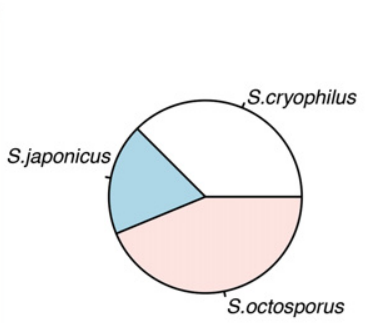
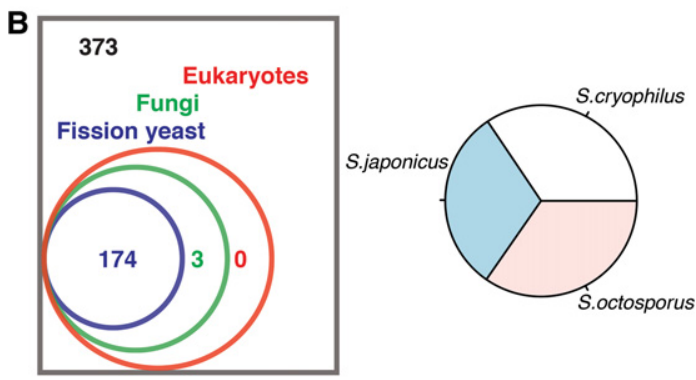

D
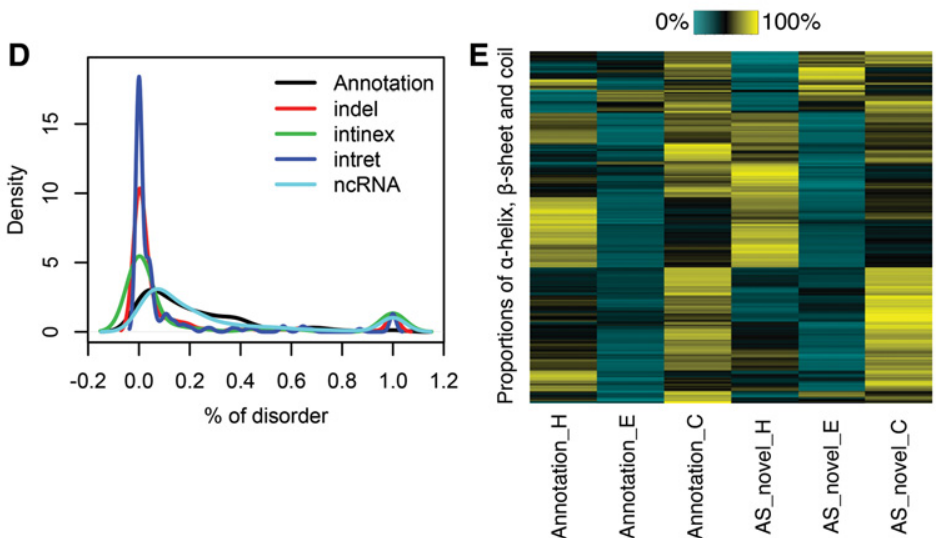

Figure 7. Conservation and secondary structure analysis. (A) Left panel shows the protein BLAST results when using the 770 novel TUs as query sequences. Numbers of novel TUs with alignment hits in different taxa are shown, where the same TU potentially has hits in multiple taxa. Right panel shows the proportion of hits in different fission yeasts. (B) Left panel shows protein BLAST results using as query sequence only the modified part of the 550 protein sequences encoded by AS isoforms. Numbers of AS isoforms with alignment hits in different taxa are shown, where the same AS isoform potentially has hits in multiple taxa. Right panel shows the proportion of hits in different fission yeasts. (C) Distribution of percent of disorder predicted by RaptorX (Källberg et al. 2012). Five classes of protein sequences were examined: full length annotated proteins, longest proteins predicted from novel TUs, $C$ terminus amino acid sequence starting at the first alteration in AS isoforms and its annotated counterpart, and longest proteins predicted from annotated ncRNA. (D) Distributions of percent of disorder predicted for annotated proteins, amino acids affected by insertions and deletions (indel), intron retentions (intret) and introns in exons (intinex) from AS isoforms, and annotated ncRNA. (E) Proportions of $\alpha$-helix (H), $\beta$-sheet (E) and coil (C) predicted from C terminus amino acid sequence starting at the first alteration in AS isoforms and its annotated counterpart.

Next, we systematically evaluated secondary structure formation of novel proteins. Annotated proteins uniformly exhibit low levels of disorder while hypothetical proteins corresponding to the longest ORFs of annotated ncRNA would have a minor mode with complete disorder (Fig. 7C). A similar bimodal pattern was observed for novel TUs and novel $\mathrm{C}$ terminus proteins from AS isoforms, as opposed to annotated $\mathrm{C}$ terminus proteins. We further explored this pattern of disordered residues among inserted (by intron retentions) and deleted (by introns in exons) amino acid sequences. We consistently observed a major mode of low disorder and a minor mode of complete disorder (Fig. 7D). Then, we asked how alternative C-terminal amino acids in AS isoforms affect secondary structures. Figure 7E and Supplemental Figure S7, D and E suggest that the majority of altered $\mathrm{C}$ termini maintain the proportion of $\alpha$-helix (H), $\beta$-sheet (E). and coil (C).

\section{Discussion}

In summary, we systematically characterized the transcriptome diversity and dynamics at the isoform level during fission yeast meiosis. We identified thousands of novel isoforms and AS events, including unexpected findings such as novel TUs and readthrough transcripts. We further carefully evaluated the functional relevance of AS isoforms by scrutinizing three factors-the abundance of AS isoforms, the differential regulation of annotated and AS isoforms during meiosis, and the translational capacity and quality of AS isoforms. The majority of AS isoforms show similar temporal patterns compared to the annotated isoforms, and the majority of AS isoforms also have shifted reading frames and short predicted protein sequences. However, a few genes show anti-correlated abundance of annotated versus AS isoforms, and these AS isoforms have similar length of translatable sequences, suggesting the possibility of AS-mediated regulation of gene activity in the unicellular organism. We developed SpliceHunter, a novel computational tool for the discovery of AS events and isoformbased sequence analysis from PacBio long sequencing reads. SpliceHunter can process single or multiple samples (e.g., time series). Our results show that the analysis of isoform dynamics is feasible using PacBio sequencing given a small transcriptome and smooth trends.

How complex is the "functional" transcriptome in $S$. pombe? In agreement with previous studies (Awan et al. 2013; Bitton et al. 2015; Stepankiw et al. 2015), our study shows that AS events are widespread in this unicellular organism. Could it be even more complex than this? Although we captured all AS events identified from prior ribosomal profiling data in meiosis, our AS events had a relatively small overlap with the AS events identified by lariat sequencing performed in log phase, diauxic shift, and heat stress (Fig. 4F). "Constitutive" AS events were detected under different conditions by different techniques and seem unlikely to be random splicing errors. AS events observed in a single condition, on the other hand, potentially include functional, condition-specific 
cases of AS. Additionally, we focused on isoforms with alternative internal structures, given the limitation of our cDNA library preparation strategy in capturing $5^{\prime}$ intact mRNAs. The diversity of AS increased substantially when we considered isoforms with precise $5^{\prime}$ and $3^{\prime}$ ends different from the annotated ones. Given the differences in AS events between the two studies, a substantially larger diversity of isoforms might be revealed in this unicellular organism when examining additional extrinsic factors like different treatments and environments. On the other hand, in plants and animals, AS events can vary across different tissues or organs. Therefore, it might be interesting to explore AS events in fission yeast with varied "developmental" status besides mitosis and meiosis, such as in young vs. aged cells or mother vs. daughter cells.

Is an isoform truly functional or just a result of splicing errors? This is a critically important question transcending the overall complexity of detected isoforms. Multiple studies have suggested that a majority of AS events are aberrant and not functional despite their prevalence (Bitton et al. 2015; Stepankiw et al. 2015). This hypothesis is supported by (1) the small number of reads supporting AS events compared to the number of reads supporting annotated splicing, (2) an increased prevalence of AS events in mutants of RNA surveillance, and (3) AS events failing to lead to meaningful translational products and ribosomes that are unlikely to be bound (Bitton et al. 2015). Our study is in line with this conclusion from the isoform perspective. Most genes have $>90 \%$ reads supporting annotated isoform (Fig. 4D); the majority of AS isoforms lead to frameshifts and shorter than annotated protein sequences (Fig. 6C-E), although we describe several interesting exceptions. We additionally show that a majority of genes have correlated abundance between annotated and AS isoform during meiosis, suggesting a lack of regulated AS from the temporal perspective for these genes. Combining all these characteristics, we suspect that a large portion of the novel isoforms are not translationally active. However, the possibility of functional AS is not excluded and could be condition-specific. AS in the alp41 and qcr10 genes were specifically induced by heat or cold shock (Awan et al. 2013).

On the other hand, we find candidate novel transcripts that are likely to be functional. For example, we identified a few hundred genes that have less reads assigned to the annotated isoforms than to the AS isoforms as described here. Moreover, certain AS isoforms were dynamically regulated during meiosis, and some of them even exhibited anti-correlated expression patterns compared to corresponding annotated isoforms (Supplemental Fig. S5). Despite the lack of evidence for translation of novel AS isoforms, we suspect that AS could explain the regulation of some of these genes in meiosis. Furthermore, the combination of conservation analysis and secondary structure modeling indicates that the predicted proteins are potentially able to form stable structures. We infer the functions of some potential novel proteins through their homologs in other related organisms. Future studies are needed to confirm the existence of predicted proteins and diagnose potential physiological functions. Additionally, AS isoforms could play regulatory, noncoding roles, similar to what was observed for some of the antisense isoforms. Finally, the fact that many novel TUs encode protein isoforms not found in closely related species could represent simply a low level of "background noise" arising from aberrant splicing, but it could also represent a mechanism for the evolution of new gene function; some subset of these reading frames may represent species-specific new gene isoform birth (Carvunis et al. 2012).

Overall, these data indicate that fission yeast has a large repertoire of AS events. Although the majority may not function to produce altered proteins, some of these may form a kind of substrate for the evolution of condition-specific isoforms. The remainder could also serve as a resource for neo-functionalization or new isoform birth. Finally, this data set will be a valuable resource for studying AS as a regulatory mechanism for a given gene of interest in this widely used model organism.

\section{Methods}

\section{General methods}

Standard methods were used for growth and meiosis of $S$. pombe. RNA extraction and library preparation are described in Supplemental Materials. Iso-Seq pre-processing was performed on the SMRT portal website. We developed software program SpliceHunter to analyze complex splicing patterns along novel isoforms inferred from Iso-Seq reads. SpliceHunter is described in more detail below. $\mathrm{R}$ (R Core Team 2015) was used for downstream data analysis and plotting, for which we provide more details in Supplemental Materials.

\section{Alternative splicing detection by SpliceHunter}

Initially (step 1 in Fig. 1A), reads are mapped to a reference genome and assigned to annotated genes (Wood et al. 2012) based on matching introns, matching splice sites, or exonic overlap, in this order. If a read does not span any introns or no splice site matches any annotated splice site, the exonic overlap with annotated genes is considered. If the read alignment overlaps multiple genes, the gene with maximal overlap is chosen, provided that the overlap is significantly larger (default $1.5 \times$ ) than the second largest overlap. Reads that do not overlap any annotated gene are used to infer novel TUs. Ambiguous reads that overlap multiple genes equally well (less than $1.5 \times$ difference) were excluded from further analysis.

For each gene and novel TU, all assigned reads are clustered to isoforms by their intron chains and their start and end sites (step 2 in Fig. 1A). Single-exons reads are grouped by the (potentially empty) sequence of retained annotated introns instead. While intron chains and retained introns are required to match perfectly, start and end sites of reads in the same cluster must lie within a sliding window of adjustable size. The start and end site of the isoform representing such a cluster is defined to be the most $5^{\prime}$ start and most $3^{\prime}$ end site, respectively, of any read contained in the cluster. For annotated genes, start and end sites of assigned reads that lie close (default $50 \mathrm{bp}$ ) to the annotated start or end site, respectively, are snapped to the annotated sites. Single-exon reads that were not assigned to an annotated gene are grouped to novel TUs simply based on their overlap.

Third (Fig. 1A, step 3), each isoform (sense) is compared to the exon-intron structure of the gene to which it was assigned to detect alternative splicing events (Supplemental Materials, Definitions 18). Single-exon isoforms (sense) are tested for intron retention events only. Start and end sites of isoforms that do not show any alternative splicing are annotated as truncated, novel (extends beyond the annotated site), or as annotated. Antisense transcripts are marked in the output as such. Isoforms whose introns match introns of multiple genes on the same strand are output as readthrough transcripts. If the matching genes lie on different strands or different chromosomes, the isoform is reported as a fused RNA molecule.

Finally, the effect of alternative splicing events on the protein sequence is studied (step 4 in Fig. 1A). Each (truncated) isoform that has been assigned to an annotated gene is extended on both ends, consistent with the exon-intron structure of the

\section{Genome Research}

www.genome.org 
annotated transcript. If the start or end site of the isoform falls into an intron of the annotated transcript, the isoform cannot be extended in $5^{\prime}$ or $3^{\prime}$ direction, respectively. If the (extended) isoform stretches beyond the position of the annotated start codon, the isoform is translated starting at the annotated start codon. For all isoforms whose structures imply an open reading frame, the corresponding protein sequences are output in FASTA format. For all remaining isoforms, the negative translation status is marked accordingly. For novel TUs, the longest ORF is reported.

Furthermore, for each isoform all alternative splicing events are annotated with the number of inserted or deleted amino acids and the resulting shift in reading frame. SpliceHunter aligns novel protein sequences with corresponding annotated sequences and outputs a sequence of strings with pattern $T x_{-} y^{\wedge} z$. $T$ is either " $\mathrm{D}$ " or "I" and indicates whether the alignment gap is a deletion (" $\mathrm{D}$ ") or insertion (" $\mathrm{I}$ "), $\mathrm{x}$ gives the length of the gap, $\mathrm{y}$ the position with respect to the reference sequence, and $z$ the frame $(0,1,2)$ following the current gap. At the end of the string, $=\mathrm{d}+/-$ denotes the positive or negative distance of the active stop codon from the annotated stop codon.

When provided with samples from multiple replicates across different time points, SpliceHunter pools all samples to cluster reads to isoforms (see second step) but keeps track of the distribution of supporting FL CCS reads across time points (see 8th column in Supplemental Table S1) or (optionally) across individual samples. SpliceHunter is also able to provide certificates for each predicted isoform. Certificates list, separately for each time point, all Iso-Seq read names from the input alignment (.bam) files that are contained in the cluster of an isoform (see second step). These certificates can be used to extract from the input .bam files the Iso-Seq reads that define an isoform across different time points and to visualize its dynamics as we illustrate for two examples in Supplemental Figure S8.

\section{Software availability}

SpliceHunter is free open-source software released under the GNU GPL license and has been developed and tested on a Linux x86_64 system. SpliceHunter's source is available as Supplemental Material and at https://bitbucket.org/canzar/splicehunter.

\section{Data access}

PacBio sequencing data from this study have been submitted to the NCBI Gene Expression Omnibus (GEO; http://www.ncbi.nlm.nih. gov/geo/) under accession number GSE79802.

\section{Acknowledgments}

We thank Amar Klar for the $S$. pombe strains. We thank Haiping Hao for assistance with sequencing, and Alix Kieu and Roberto Lleras, Pacific Biosciences, for in-kind contribution of SMRT cells and suggestions for analysis. We thank Ed Miller for providing funding to the High Throughput Biology Center to acquire the PacBio sequencer, and Steven Salzberg and Kai Kammers for helpful discussions.

\section{References}

Au KF, Sebastiano V, Afshar PT, Durruthy JD, Lee L, Williams BA, van Bakel H, Schadt EE, Reijo-Pera RA, Underwood JG, et al. 2013. Characterization of the human ESC transcriptome by hybrid sequencing. Proc Natl Acad Sci 110: E4821-E4830.

Awan AR, Manfredo A, Pleiss JA. 2013. Lariat sequencing in a unicellular yeast identifies regulated alternative splicing of exons that are evolutionarily conserved with humans. Proc Natl Acad Sci 110: 12762-12767.
Bentley DL. 2014. Coupling mRNA processing with transcription in time and space. Nat Rev Genet 15: 163-175.

Bitton DA, Atkinson SR, Rallis C, Smith GC, Ellis DA, Chen YY, Malecki M, Codlin S, Lemay JF, Cotobal C, et al. 2015. Widespread exon skipping triggers degradation by nuclear RNA surveillance in fission yeast. Genome Res 25: 884-896.

Braunschweig U, Gueroussov S, Plocik AM, Graveley BR, Blencowe BJ. 2013. Dynamic integration of splicing within gene regulatory pathways. Cell 152: $1252-1269$.

Carvunis A, Rolland T, Wapinski I, Calderwood MA, Yildirim MA, Simonis N, Charloteaux B, Hidalgo CA, Barbette J, Santhanam B. 2012. Protogenes and de novo gene birth. Nature 487: 370-374.

Costa FF. 2007. Non-coding RNAs: lost in translation? Gene 386: 1-10.

Dong L, Liu H, Zhang J, Yang S, Kong G, Chu JS, Chen N, Wang D. 2015. Single-molecule real-time transcript sequencing facilitates common wheat genome annotation and grain transcriptome research. BMC Genomics 16: 1039.

Duncan CD, Mata J. 2014. The translational landscape of fission-yeast meiosis and sporulation. Nat Struct Mol Biol 21: 641-647.

Eid J, Fehr A, Gray J, Luong K, Lyle J, Otto G, Peluso P, Rank D, Baybayan P, Bettman B, et al. 2009. Real-time DNA sequencing from single polymerase molecules. Science 323: 133-138.

Eser P, Wachutka L, Maier KC, Demel C, Boroni M, Iyer S, Cramer P, Gagneur J. 2016. Determinants of RNA metabolism in the Schizosaccharomyces pombe genome. Mol Syst Biol 12: 857.

Gish W, States DJ. 1993. Identification of protein coding regions by database similarity search. Nat Genet 3: 266-272.

Gordon SP, Tseng E, Salamov A, Zhang J, Meng X, Zhao Z, Kang D, Underwood J, Grigoriev IV, Figueroa M. 2015. Widespread polycistronic transcripts in fungi revealed by single-molecule mRNA sequencing. PLoS One 10: e0132628.

Hüttenhofer A, Schattner P, Polacek N. 2005. Non-coding RNAs: hope or hype? Trends Genet 21: 289-297.

Källberg M, Wang H, Wang S, Peng J, Wang Z, Lu H, Xu J. 2012. Templatebased protein structure modeling using the RaptorX web server. Nat Protoc 7: 1511-1522.

Keren H, Lev-Maor G, Ast G. 2010. Alternative splicing and evolution: diversification, exon definition and function. Nat Rev Genet 11: 345-355.

Laufman O, Ben Yosef R, Adir N, Manor H. 2005. Cloning and characterization of the Schizosaccharomyces pombe homologs of the human protein Translin and the Translin-associated protein TRAX. Nucleic Acids Res 33: 4128-4139.

Le Hir H, Nott A, Moore MJ. 2003. How introns influence and enhance eukaryotic gene expression. Trends Biochem Sci 28: 215-220.

Lee Y, Rio DC. 2015. Mechanisms and regulation of alternative pre-mRNA splicing. Аnпu Rev Biochem 84: 291-323.

Martin JA, Johnson NV, Gross SM, Schnable J, Meng X, Wang M, ColemanDerr D, Lindquist E, Wei C, Kaeppler S. 2014. A near complete snapshot of the Zea mays seedling transcriptome revealed from ultra-deep sequencing. Sci Rep 4: 4519.

Martín-Castellanos C, Blanco M, Rozalén AE, Pérez-Hidalgo L, García AI, Conde F, Mata J, Ellermeier C, Davis L, San-Segundo P. 2005. A largescale screen in $S$. pombe identifies seven novel genes required for critical meiotic events. Curr Biol 15: 2056-2062.

Mata J, Lyne R, Burns G, Bähler J. 2002. The transcriptional program of meiosis and sporulation in fission yeast. Nat Genet 32: 143-147.

McGlincy NJ, Smith CW. 2008. Alternative splicing resulting in nonsensemediated mRNA decay: What is the meaning of nonsense? Trends Biochem Sci 33: 385-393.

Minoche AE, Dohm JC, Schneider J, Holtgräwe D, Viehöver P, Montfort M, Sörensen TR, Weisshaar B, Himmelbauer H. 2015. Exploiting singlemolecule transcript sequencing for eukaryotic gene prediction. Genome Biol 16: 184.

Mullen JR, Kayne PS, Moerschell RP, Tsunasawa S, Gribskov M, ColavitoShepanski M, Grunstein M, Sherman F, Sternglanz R. 1989. Identification and characterization of genes and mutants for an N-terminal acetyltransferase from yeast. EMBO J 8: 2067-2075.

Ni T, Tu K, Wang Z, Song S, Wu H, Xie B, Scott KC, Grewal SI, Gao Y, Zhu J. 2010. The prevalence and regulation of antisense transcripts in Schizosaccharomyces pombe. PLoS One 5: e15271.

Nilsen TW, Graveley BR. 2010. Expansion of the eukaryotic proteome by alternative splicing. Nature 463: 457-463.

R Core Team. 2015. R: a language and environment for statistical computing. $\mathrm{R}$ Foundation for Statistical Computing, Vienna, Austria. https://www.Rproject.org/.

Rhoads A, Au KF. 2015. PacBio sequencing and its applications. Genomics Proteomics Bioinformatics 13: 278-289.

Sharon D, Tilgner H, Grubert F, Snyder M. 2013. A single-molecule longread survey of the human transcriptome. Nat Biotechnol 31: 1009-1014. 
Kuang et al.

Stepankiw N, Raghavan M, Fogarty EA, Grimson A, Pleiss JA. 2015 Widespread alternative and aberrant splicing revealed by lariat sequencing. Nucleic Acids Res 43: 8488-8501.

Thomas S, Underwood JG, Tseng E, Holloway AK. 2014. Long-read sequencing of chicken transcripts and identification of new transcript isoforms. PLoS One 9: e94650.

Tilgner H, Grubert F, Sharon D, Snyder MP. 2014. Defining a personal, allele-specific, and single-molecule long-read transcriptome. Proc Natl Acad Sci 111: 9869-9874.

Tilgner H, Jahanbani F, Blauwkamp T, Moshrefi A, Jaeger E, Chen F, Harel I, Bustamante CD, Rasmussen M, Snyder MP. 2015. Comprehensive transcriptome analysis using synthetic long-read sequencing reveals molecular co-association of distant splicing events. Nat Biotechnol 33: 736-742.

Wang Z, Burge CB. 2008. Splicing regulation: from a parts list of regulatory elements to an integrated splicing code. RNA 14: 802-813.
Wang ET, Sandberg R, Luo S, Khrebtukova I, Zhang L, Mayr C, Kingsmore SF, Schroth GP, Burge CB. 2008. Alternative isoform regulation in human tissue transcriptomes. Nature 456: 470-476.

Wilhelm BT, Marguerat S, Watt S, Schubert F, Wood V, Goodhead I, Penkett CJ, Rogers J, Bähler J. 2008. Dynamic repertoire of a eukaryotic transcriptome surveyed at single-nucleotide resolution. Nature 453: 1239-1243.

Wood V, Harris MA, McDowall MD, Rutherford K, Vaughan BW, Staines DM, Aslett M, Lock A, Bahler J, Kersey PJ, et al. 2012. PomBase: a comprehensive online resource for fission yeast. Nucleic Acids Res 40: D695-D699.

Received April 8, 2016; accepted in revised form November 14, 2016.

\section{Genome Research}

www.genome.org 


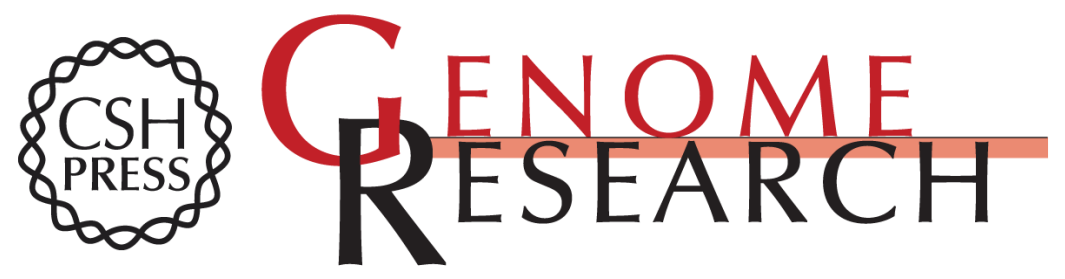

\title{
The dynamic landscape of fission yeast meiosis alternative-splice isoforms
}

\author{
Zheng Kuang, Jef D. Boeke and Stefan Canzar
}

Genome Res. 2017 27: 145-156 originally published online November 17, 2016

Access the most recent version at doi:10.1101/gr.208041.116

\section{Supplemental Material}

References

Creative

Commons

License

Email Alerting Service
http://genome.cshlp.org/content/suppl/2016/12/19/gr.208041.116.DC1

This article cites 37 articles, 7 of which can be accessed free at: http://genome.cshlp.org/content/27/1/145.full.html\#ref-list-1

This article is distributed exclusively by Cold Spring Harbor Laboratory Press for the first six months after the full-issue publication date (see

http://genome.cshlp.org/site/misc/terms.xhtml). After six months, it is available under a Creative Commons License (Attribution-NonCommercial 4.0 International), as described at http://creativecommons.org/licenses/by-nc/4.0/.

Receive free email alerts when new articles cite this article - sign up in the box at the top right corner of the article or click here.

\section{Affordable, Accurate Sequencing.}

To subscribe to Genome Research go to: https://genome.cshlp.org/subscriptions 